\title{
Combined IFN- $\alpha$ and 5-FU treatment as a postoperative adjuvant following surgery for hepatocellular carcinoma with portal venous tumor thrombus
}

\author{
HIROAKI NAGANO ${ }^{1}$, SHOGO KOBAYASHI ${ }^{1}$, SHIGERU MARUBASHI $^{1}$, HIROSHI WADA ${ }^{1}$, HIDETOSHI EGUCHI ${ }^{1}$, \\ MASAHIRO TANEMURA ${ }^{1}$, YOSHITO TOMIMARU ${ }^{1}$, KOJI UMESHITA ${ }^{2}$, YUICHIRO DOKI ${ }^{1}$ and MASAKI MORI $^{1}$ \\ Departments of ${ }^{1}$ Surgery and ${ }^{2}$ Health Science, Graduate School of Medicine, Osaka University, Osaka, Japan
}

Received April 29, 2011; Accepted November 30, 2011

DOI: 10.3892 /etm.2012.736

\begin{abstract}
The efficacy of combination therapy with subcutaneous interferon (IFN)- $\alpha$ and intra-arterial 5-fluorouracil (5-FU) as a postoperative adjuvant for resectable advanced hepatocellular carcinoma (HCC) invading the major branches of the portal vein (PVTT) was examined. The prognosis of HCC with PVTT (Vp3 or 4) is extremely poor. Recently, we reported the possibility of combination therapy with IFN- $\alpha$ and intra-arterial 5-FU for intractable HCC with PVTT as a postoperative adjuvant and this is the second report. Patients with HCC with PVTT were included $(n=50)$. Thirty consecutive patients with HCC and PVTT were treated with 3 cycles of a combination therapy consisting of arterial 5-FU infusion (300 mg/mm $3 /$ day, 5 days/week, for the initial 2 weeks) and IFN subcutaneous injection (5 MIU, 3 times/week, 4 weeks) as a postoperative adjuvant following hepatic resection; another 20 patients receiving no IFN/5-FU chemotherapy acted as controls. Results for the IFN/5-FU adjuvant treatment group were as follows: disease-free survival ( $n=9,15-109$ months), survival with recurrence ( $n=6,30-92$ months), cancer death ( $n=9,14-60$ months), death from other causes but no recurrence $(n=5,13-87$ months) and death from other causes with recurrence ( $n=1,22$ months). The 1-year survival rate was $100 \%$ in patients treated with IFN/5-FU, and $30 \%$ in those without IFN/5-FU as historical controls $(n=20)$. There was a significant difference in disease-free and overall survival rates between the two groups $(\mathrm{P}<0.0001)$. In conclusion, IFN/5-FU
\end{abstract}

Correspondence to: Dr Hiroaki Nagano, Department of Surgery, Graduate School of Medicine, Osaka University, 2-2 Yamadaoka E-2, Suita 565-0871, Osaka, Japan

E-mail: hnagano@gesurg.med.osaka-u.ac.jp

Abbreviations: HCC, hepatocellular carcinoma; IFN, interferon; AFP, $\alpha$-fetoprotein; PIVKA-II, protein induced by vitamin K antagonist or absence; PVTT, portal vein tumor thrombosis; 5-FU, 5-fluorouracil; TAE, transcatheter arterial embolization; RFA, radiofrequency ablation

Key words: HCC, PVTT, IFN, chemotherapy, surgery combination therapy may be a very promising postoperative adjuvant treatment for HCC with PVTT.

\section{Introduction}

Hepatocellular carcinoma (HCC) is a common malignancy worldwide and is now the third major cause of cancer-related death in Japan (1). Vascular invasion, particularly portal veinous tumor thrombus (PVTT), is one of the indicators of patient prognosis and has been well documented. The mortality rate is very high in patients with unresectable tumors, and the quality of life (QOL) is poor due to intractable ascites or esophageal bleeding. In such a situation, conventional therapies generally have no clinical effect, therefore, a new strategy is required for patients of advanced HCC with PVTT in the major trunk.

Recently, sorafenib, an oral multikinase inhibitor of the vascular endothelial growth factor receptor, the plateletderived growth factor receptor and Raf, has been demonstrated to prolong median survival and the time to progression by nearly 3 months in patients with advanced HCC as compared with those administered a placebo (2). However, no complete response and few partial responses $(2 \%)$ were found in the same study. Although this drug can be used for the treatment of patients with advanced HCC, its clinical effectiveness is still controversial in Japan. According to the consensus-based clinical manual proposed by the Japan Society of Hepatology (3), arterial infusion chemotherapy using an implantable drug delivery system is recommended as one of the treatments for advanced HCC with portal venous invasion. Several recent studies have indicated the beneficial effects of interferon (IFN)- $\alpha$-based combination chemotherapies for HCC (4-8). We also reported the clinical efficiency of IFN- $\alpha$ and 5-fluorouracil (5-FU) combination therapy for advanced HCC with portal venous tumor thrombi and intrahepatic metastasis (9-11), including the mechanism of the anti-tumor effect (12-19). In addition, we applied this combined chemotherapy (IFN/5-FU) for resectable HCC as a postoperative adjuvant (20) and a multimodal treatment (21).

In the present study, we investigated the clinical effect of IFN/5-FU therapy for resectable advanced HCC with PVTT as a postoperative adjuvant therapy, as an extention of our previous report (20). 


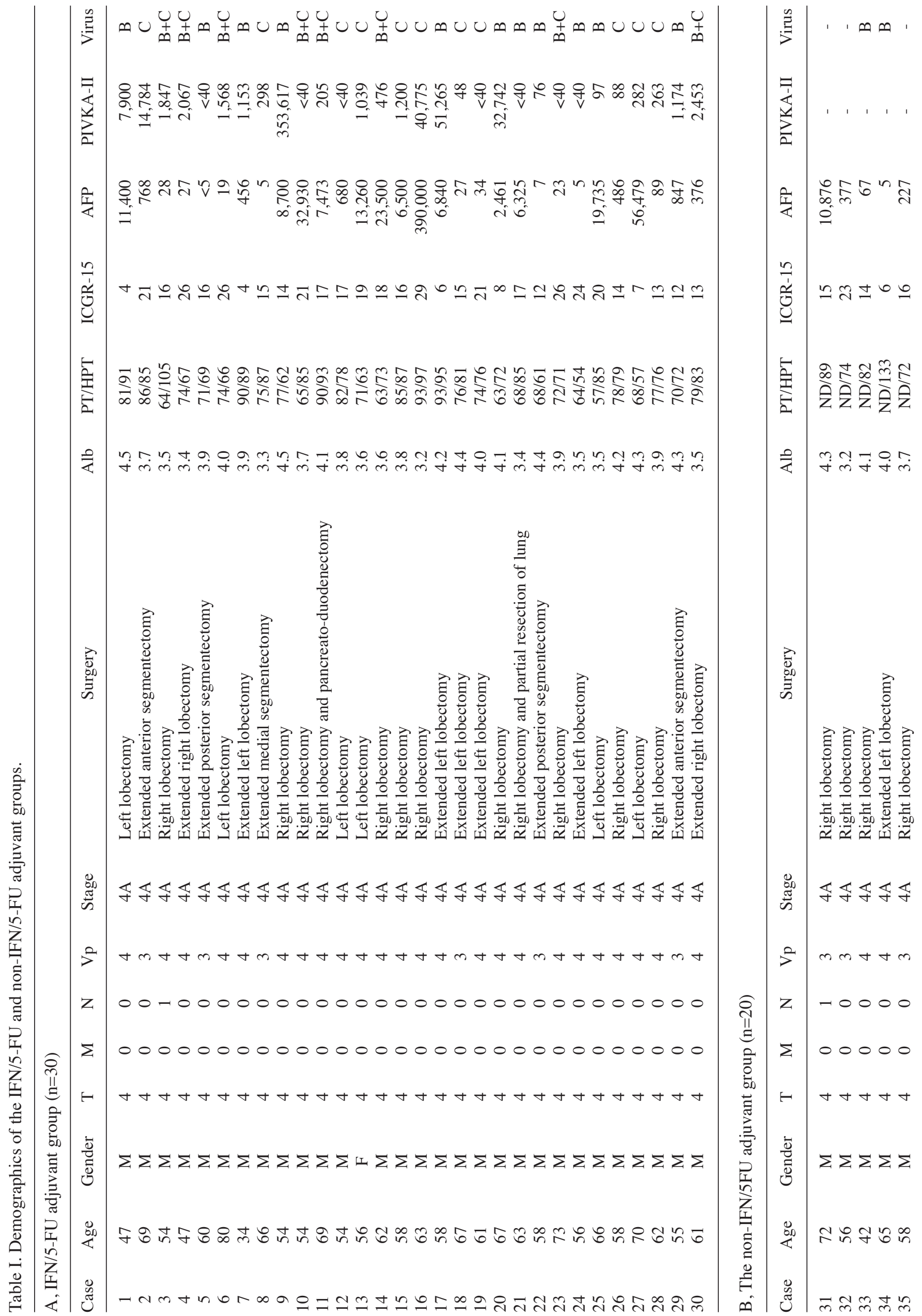




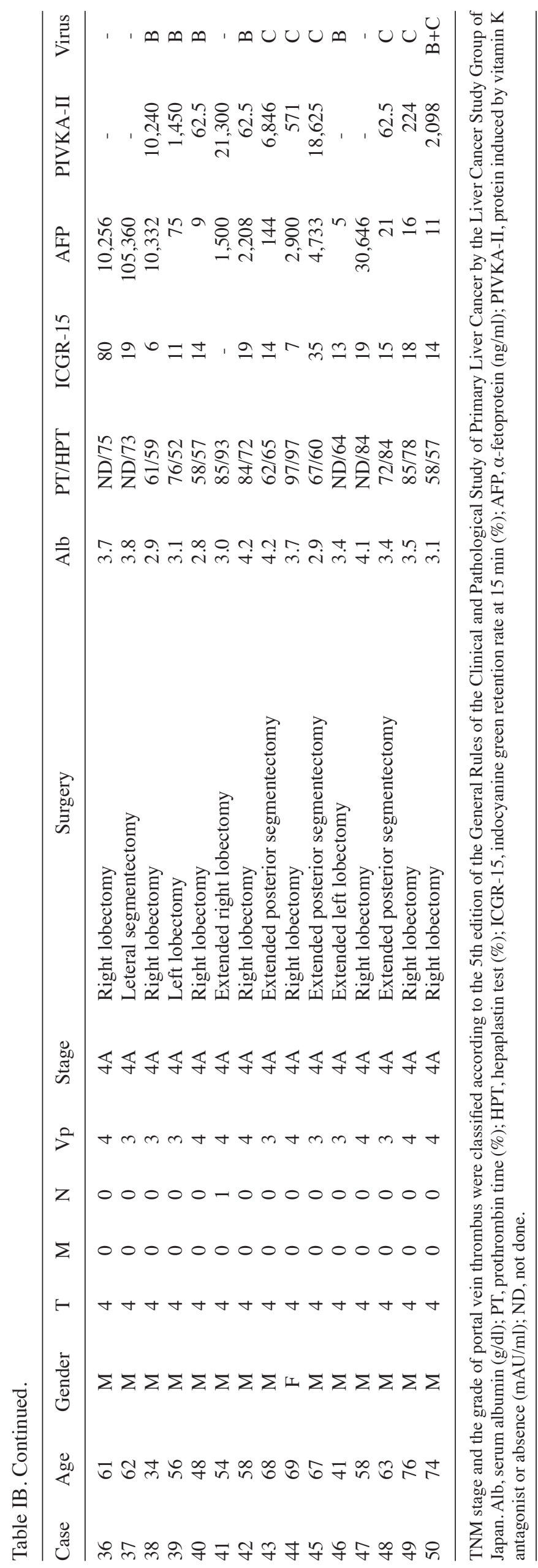

\section{Patients and methods}

Patients. Of the patients with HCC who were admitted and underwent curative hepatic resection at the Department of Surgery, Osaka University Hospital, 50 were included in this study based on the identification of a tumor thrombus either in the major or first branch of the portal vein ( $\mathrm{Vp} 3$ or 4) (22). Liver function tests and imaging techniques, including computed tomography (CT) with hepatic angiography and arterial portography, revealed that these cases were resectable and subsequently they underwent hepatectomy. Of the 50 patients, 30 patients, from 1998 to 2008, had an intra-arterial catheter inserted through the gastro-duodenal artery with an implanted drug delivery system during the operation to facilitate postoperative adjuvant IFN/5-FU combined chemotherapy (9-11). They were treated with 3 cycles as a postoperative adjuvant. The demographic data of these patients are shown in Table IA. The 15 patients, no. 1-15, are the same patients as in our previous study (20), followed up for a longer period. Another 20 patients, from 1987 to 2007, with the same tumor stage of advanced HCC and $\mathrm{Vp} 3$ or 4 , underwent surgery but did not receive combined IFN/5-FU therapy. They were treated with appropriate local HCC therapy except for 3 cases (no. 44, 45 and 48) who received only 5-FU intra-arterial chemotherapy without IFN. The demographic data of these patients are shown in Table IB. The 15 patients, no. 31-45, are the same patients as in our previous study (28), followed up for a longer period. The cases, no. 31-43 are the historical controls; no. 44-50 refused IFN/5-FU combined chemotherapy. These 2 groups were compared in terms of features of HCC, hepatic function, surgery, clinical effects, disease-free and overall survival.

The TNM stage and grade of portal vein thrombus were classified according to the 5th edition of the General Rules for the Clinical and Pathological Study of Primary Liver Cancer by the Liver Cancer Study Group of Japan (22). The criteria for selection for intra-arterial combination treatment included i) the absence of extra-hepatic metastases, ii) AST and ALT levels $<100 \mathrm{IU} / 1$, iii) a platelet count $>80,000 / \mathrm{mm}^{3}$, iv) successful implantation of an intra-arterial catheter and drug delivery system and v) a performance status (Eastern Cooperative Oncology Group, ECOG) (23) of level 0-1.

Treatment regimen of IFN/5-FU combination chemotherapy and follow-up after surgery. After obtaining informed written consent, each patient was treated with subcutaneous administration of IFN- $\alpha$ (OIF, Otsuka Pharmaceutical Co., Tokyo) and an intra-arterial infusion of 5-FU (Kyowa Hakko Co., Tokyo). IFN- $\alpha$ [5x106 U (5 MU)] was administered on Days 1,3 and 5 of every week (9-11). Continuous infusion chemotherapy (5-FU, $300 \mathrm{mg} / \mathrm{mm}^{3} /$ day) through the proper hepatic artery was applied 5 days/week for 2 weeks via a catheter connected to a subcutaneously implanted drug delivery system. All anti-cancer therapies were discontinued when adverse effects reached level 2 according to the ECOG classification (23). In addition to serum chemistry, tumor markers such as $\alpha$-fetoprotein (AFP) and protein induced by vitamin K antagonist or absence (PIVKA-II) were measured at least once every one month. An abdominal CT scan or dynamic magnetic resonance imaging (MRI) was also performed at least once every 3 months following surgery. 


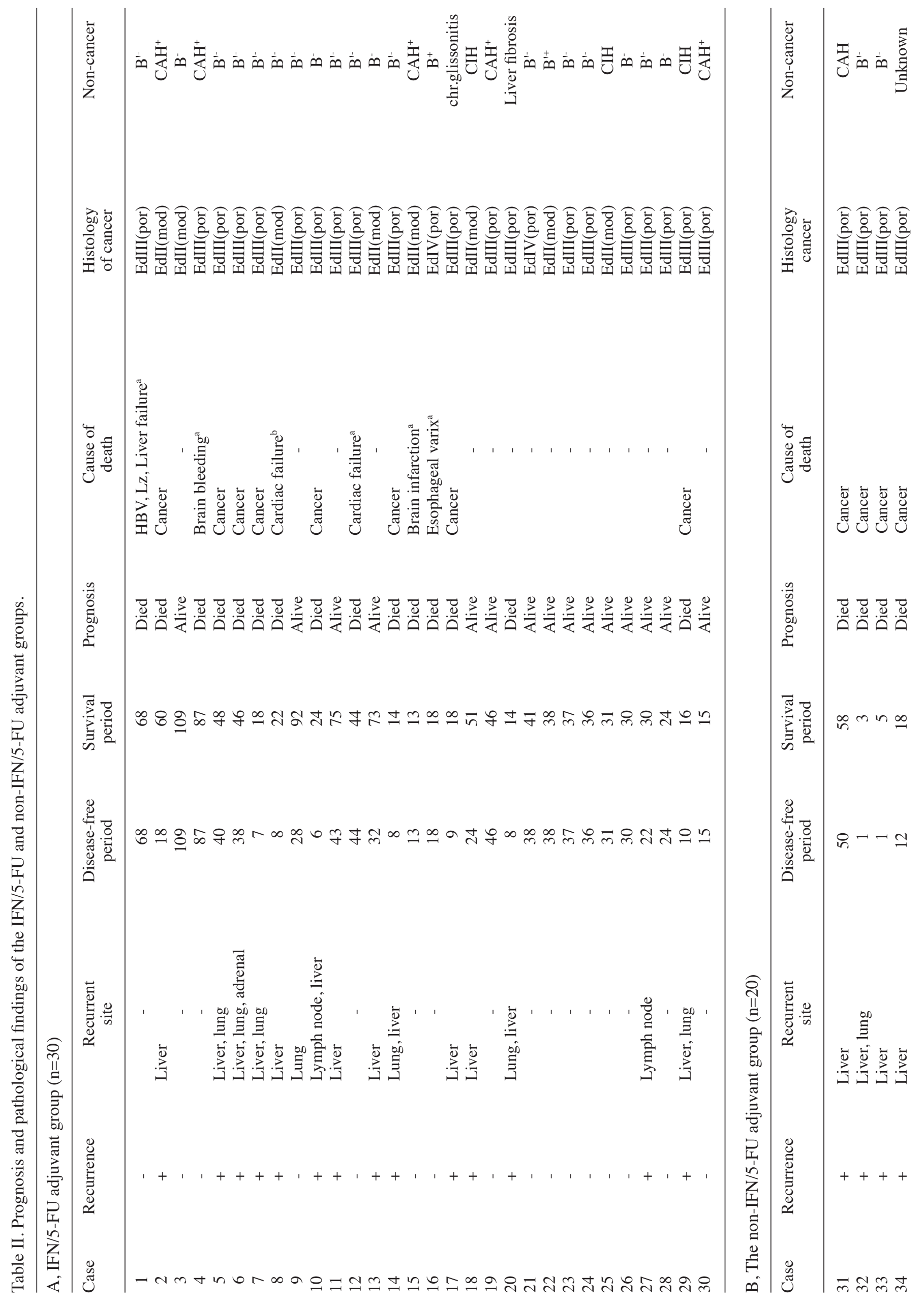




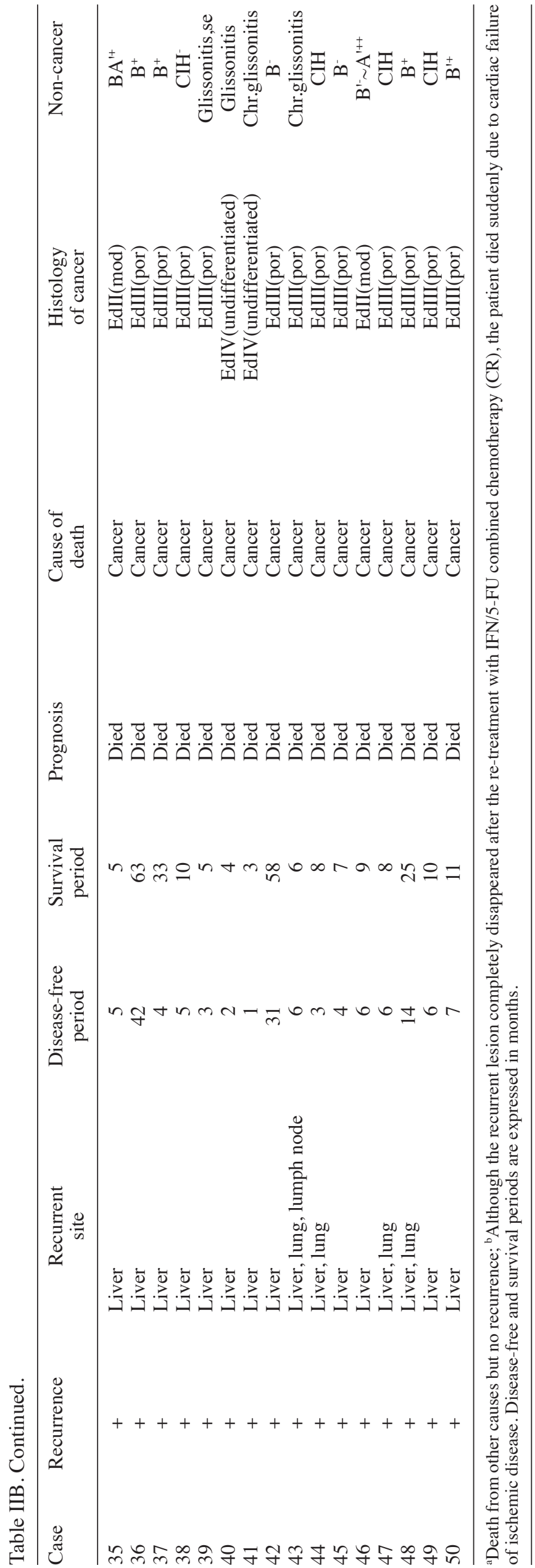

Statistical analysis. Survival curves were constructed using the Kaplan-Meier method (24). Survival curves were compared using the log-rank test. The features of HCC, biochemistry, ICGR-15, and virus status were compared using the MannWhitney test. The level of tumor markers (AFP and PIVKA-II) was compared by the Wilcoxon matched-pair test. Statistical significance was interpreted as $\mathrm{P}<0.05$.

\section{Results}

Features of the preoperative hepatic function, hepatocellular carcinoma and surgery. The features of the preoperative hepatic function are shown in Table I. There was no significant difference between the IFN/5-FU adjuvant and non-IFN/5-FU adjuvant groups in terms of the preoperative hepatic function: serum albumin $(\mathrm{g} / \mathrm{dl})$, prothrombin time (PT, \%), hepaplastin test (HPT, \%), indocyanine green retention rate at $15 \mathrm{~min}$ (ICGR-15, \%). No difference was also demonstrated in terms of tumor stage, surgical procedure, including AFP $(\mathrm{ng} / \mathrm{ml})$ and PIVKA-II (mAU/ml) (Table I).

Clinical effects, disease-free and overall survival. Concerning the enrolled 50 patients, none developed any major complications. The $30 \mathrm{IFN} / 5-\mathrm{FU}$ patients started the postoperative adjuvant therapy 3-5 weeks after surgery and completed 3 cycles of treatment. In addition, the QOL of patients in this study was excellent, as this adjuvant therapy was performed at outpatient clinics; no hospital admission was necessary. The patients were able to maintain their social life while receiving IFN/5-FU adjuvant therapy.

The follow-up period of the present study was from 3 to 109 months (mean 24 months). The difference in follow-up was significant compared to the prior study (20). Results for the IFN/5-FU adjuvant treatment group were as follows: diseasefree survival ( $\mathrm{n}=9)$ (15-109 months), survival with recurrence $(n=6)(30-92$ months), cancer death ( $n=9)(14-60$ months), death from other causes but no recurrence $(n=5)$ (13-87 months) and death from other causes with recurrence ( $n=1,22$ months). The summary of these results for each case is shown in Table IIA.

In the group that received no adjuvant IFN/5-FU therapy, 14 of 20 patients died of recurrent cancer within 1 year; almost all patients (17 of 20) within 3 years. All patients developed recurrences in the residual liver, 4 also had lung metastasis, and one had lung and lymph node metastases. Recurrence was identified within 1 year after hepatic resection in 16 of the 20 patients. These clinical results are summarized in Table IIB.

With respect to survival, the 1-, 3- and 5-year disease-free survival rates were 77,60 and $39 \%$ for patients who received IFN/5-FU combination therapy $(\mathrm{n}=30) ; 20,10$ and $0 \%$, respectively, for the historical controls $(\mathrm{n}=20)$ (Fig. 1A). In addition, the 1-, 3- and 5-year overall survival rates were 100, 69 and 44\% for patients who received IFN/5-FU combination therapy $(n=30) ; 30,15$ and 5\% for the historical controls $(n=20)$ (Fig. 1B). There was a significant difference in disease-free and overall survival rates between these two groups (disease-free, $\mathrm{P}<0.0001$; overall, $\mathrm{P}<0.0001$ ) (Fig. 1).

Adverse effects. No leukopenia, thrombocytopenia, or myelosuppression was observed in the 30 patients of the IFN/5-FU group. Other adverse effects were, in general, clinically 
A

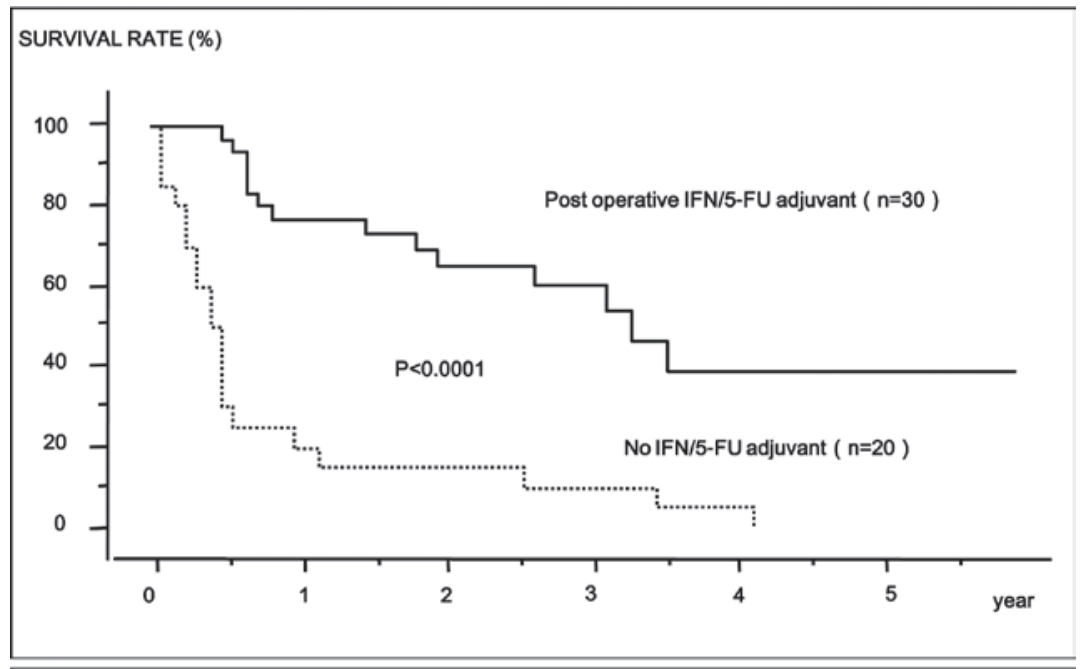

B

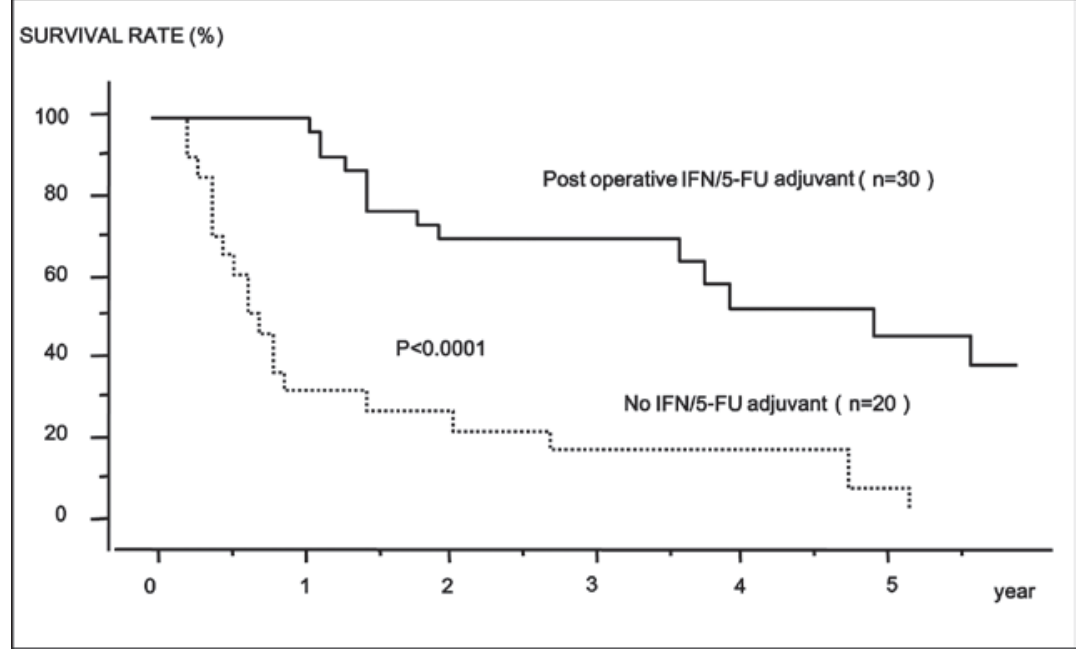

Figure 1. (A) Disease-free survival rates of patients grouped according to whether they received IFN/5-FU combined chemotherapy or not as a postoperative adjuvant following hepatic resection. A statistically significant difference in survival was noted $(\mathrm{P}<0.0001)$. (B) Overall survival rates of patients grouped according to whether they received IFN/5-FU combined chemotherapy or not as a postoperative adjuvant following hepatic resection. A statistically significant difference in survival was noted $(\mathrm{P}=0.0001)$.

manageable. Fever was commonly observed but was easily controlled by non-steroidal anti-inflammatory drugs prior to IFN injection. No depression due to IFN administration was observed in any of the 30 patients.

\section{Discussion}

The present study is the extended examination of our previous report (20) concerning the clinical outcome of a combination therapy of IFN- $\alpha$ and 5-FU as a postoperative adjuvant therapy for resectable HCC with PVTT. Our results showed that this treatment regimen markedly decreased the incidence of recurrence in the residual liver and significantly prolonged the disease-free and overall survival periods compared with historical controls. Amazingly, the 1-year overall survival rate was $100 \%$ in the IFN/5-FU treatment group. This result concerning the survival benefit for resectable far advanced HCC with PVTT was much better than the Japan survey for HCC patients (25).

Development of tumor thrombi in a major branch or main trunk of the portal vein is a frequent terminal feature of $\mathrm{HCC}$, either with primary or recurrent tumors. The prognosis of such patients is extremely poor, and survival is limited to a few months after diagnosis (26-31). For these advanced HCCs, conventional therapies like transcatheter arterial embolization (TAE) and radiofrequency ablation (RFA) are not indicated due to lack of efficacy and associated complications (30-32). Liver transplantation is a contra-indication for such far advanced HCC with PVTT cases (33). To date, several reports have mentioned the feasibility of hepatic resection for patients with PVTT, however the outcome is unsatisfactory $(28,29,34,35)$, except for limited cases with PVTT located in the segmental or sectoral branches (36). Based on this finding, in the absence of effective preoperative and/or postoperative adjuvant therapy, hepatic resection should not be offered in cases with PVTT in the main trunk or first branch. Several approaches have been attempted to improve the surgical results, including radiotherapy and TAE (37-40). Compared with these reports, our clinical outcome using IFN/5-FU combined therapy as a postoperative adjuvant was excellent and highly satisfactory, in terms of survival and long-term outcome.

In regards to recurrence, extrahepatic metastases often occurred following surgery even after IFN/5-FU adjuvant treatment. The combination of IFN/5-FU is not effective 
against extrahepatic metastases. This is understandable as 5-FU, administered into the hepatic artery, does not reach extrahepatic tissues at a high concentration. In such a situation, systemic administration of 5-FU or related agents may be effective against extrahepatic lesions in combination with IFN- $\alpha$ (41). This possibility is highly interesting since the implantation of a dwelling catheter is one of the demerits of the present combination therapy (9-11). Recently, several molecular-targeting agents have been developed and applied for HCC treatment (42-44). Particularly, sorafenib is the first agent leading to improved overall survival for patients with advanced HCC, as revealed in a phase III clinical trial (10). These molecular-targeting agents are a very effective therapeutic modality, which exhibited a different mechanism of anti-tumor effect compared to IFN/5-FU combination as an cytotoxic medicine. Based on this evidence, mutual interaction and shared roles may be extremely important for the progression of treatment for intractable advanced HCC. We previously reported that PTK $/ \mathrm{ZK}$, a type of molecular-targeting medicine, enhanced the anti-tumor effect of IFN/5-FU in vitro (45).

Myelosuppressive adverse effects frequently occur in patients with HCC. This is not only because thrombocytopenia and/or leukopenia are often present prior to the initiation of anti-cancer therapy, but because treatment often has to be discontinued due to these side effects. Another advantage of our combination therapy was the markedly low incidence of myelosuppressive side effects; no patient developed leukopenia in the present study (data not shown). In addition, the QOL of patients in the present study was excellent, as IFN/5-FU adjuvant therapy was performed at outpatient clinics. No hospital admission was necessary for the administration of the IFN injection combined with the intra-arterial perfusion chemotherapy. The patients were able to maintain their social life while receiving IFN/5-FU adjuvant therapy. Moreover, they had no symptoms related to liver dysfunction.

In conclusion, this study indicated that combination chemotherapy with subcutaneous IFN- $\alpha$ and intra-arterial 5-FU is a promising strategy for resectable advanced HCC with PVTT in the main trunk or first branch, as a postoperative adjuvant therapy following surgery.

\section{Acknowledgements}

This study was supported by a grant in aid for cancer research from the Ministry of Health and Walfare and the Ministry of Culture and Science in Japan.

\section{References}

1. Arii S, Monden K, Niwano M, et al: Results of surgical treatment for recurrent hepatocellular carcinoma; comparison of outcome among patients with multicentric carcinogenesis, intrahepatic metastasis, and extrahepatic recurrence. J Hepatobiliary Pancreat Surg 5: 86-92, 1998.

2. Llovet JM, Ricci S, Mazzaferro V, et al: Sorafenib in advanced hepatocellular carcinoma. N Engl J Med 359: 378-390, 2008.

3. Kudo M and Okanoue T: Management of hepatocellular carcinoma in Japan: consensus-based clinical practice manual proposed by the Japan Society of Hepatology. Oncology 72 (Suppl 1): S2-S15, 2007.

4. Chung YH, Song IH, Song BC, et al: Combined therapy consisting of intraarterial cisplatin infusion and systemic interferon-alpha for hepatocellular carcinoma patients with major portal vein thrombosis or distant metastasis. Cancer 88: 1986-1991, 2000.
5. Kaneko S, Urabe T and Kobayashi S: Combination chemotherapy for advanced hepatocellular carcinoma complicated by major portal vein thrombosis. Oncology 62 (Suppl 1): S69-S73, 2002.

6. Patt YZ, Hassan MM, Lozano RD, et al: Phase II trial of systemic continuous fluorouracil and subcutaneous recombinant interferon Alfa-2b for treatment of hepatocellular carcinoma. J Clin Oncol 21: 421-427, 2003.

7. Obi S, Yoshida H, Toune R, et al: Combination therapy of intraarterial 5-fluorouracil and systemic interferon-alpha for advanced hepatocellular carcinoma with portal venous invasion. Cancer 106: 1990-1997, 2006.

8. Uka K, Aikata H, Takaki S, et al: Similar effects of recombinant interferon-alpha-2b and natural interferon-alpha when combined with intra-arterial 5-fluorouracil for the treatment of advanced hepatocellular carcinoma. Liver Int 27: 1209-1216, 2007.

9. Sakon M, Nagano H, Dono K, et al: Combined intraarterial 5-fluorouracil and subcutaneous interferon- $\alpha$ therapy for advanced hepatocellular carcinoma with tumor thrombi in the major portal branches. Cancer 94: 435-442, 2002.

10. Ota H, Nagano H, Sakon M, et al: Treatment of hepatocellular carcinoma with major portal vein thrombosis by combined therapy with subcutaneous interferon-alpha and intra-arterial 5-fluorouracil; role of type 1 interferon receptor expression. Br J Cancer 93: 57-64, 2005.

11. Nagano H, Wada H, Kobayashi S, et al: Long-term outcome of combined subcutaneous interferon- $\alpha$ and intra-arterial 5-fluorouracil treatment for advanced hepatocellular carcinoma with major portal vein thrombosis. Oncology (In Press).

12. Eguchi H, Nagano H, Yamamoto $\mathrm{H}$, et al: Augmentation of antitumor activity of 5-fluorouracil by interferon alpha is associated with up-regulation of p27Kipl in human hepatocellular carcinoma cells. Clin Cancer Res 6: 2881-2890, 2000

13. Yamamoto T, Nagano H, Sakon M, et al: Partial contribution of tumor necrosis factor-related apoptosis-inducing ligand (TRAIL)/TRAIL receptor pathway to anti-tumor effects of interferon-alpha/5-fluorouracil against hepatocellular carcinoma. Clin Cancer Res 10: 7884-7895, 2004.

14. Kondo M, Nagano H, Wada H, et al: Combination of IFN-alpha and 5-fluorouracil induces apoptosis through IFN-alpha/beta receptor in human hepatocellular carcinoma cells. Clin Cancer Res 11: 1277-1286, 2005.

15. Nakamura M, Nagano H, Sakon M, et al: Role of the Fas/FasL pathway in combination therapy with interferon-alpha and fluorouracil against hepatocellular carcinoma in vitro. J Hepatol 46: 77-88, 2007.

16. Noda T, Nagano H, Takemasa I, et al: Activation of Wnt/ beta-catenin signalling pathway induces chemoresistance to interferon-alpha/5-fluorouracil combination therapy for hepatocellular carcinoma. Br J Cancer 100: 1647-1658, 2009.

17. Wada $\mathrm{H}$, Nagano $\mathrm{H}$, Yamamoto $\mathrm{H}$, et al: Combination of interferon-alpha and 5-fluorouracil inhibits endothelial cell growth directly and by regulation of angiogenic factors released by tumor cells. BMC Cancer 9: 361-372, 2009.

18. Tomimaru Y, Eguchi H, Wada H, et al: Insulin-like growth factor-binding protein 7 alters the aensitivity to interferon-based anticancer therapy in hepatocellular carcinoma cells. Br J Cancer 102: 1483-1490, 2010.

19. Tomimaru Y, Eguchi H, Nagano H, et al: MicroRNA-21 induces resistance to the anti-tumor effect of interferon- $\alpha / 5$-fluorouracil in hepatocellular carcinoma cells. Br J Cancer 103: 1617-1626, 2010.

20. Nagano H, Sakon M, Eguchi H, et al: Hepatic resection followed by IFN-alpha and 5-FU for advanced hepatocellular carcinoma with tumor thrombus in the major portal branch. Hepatogastroenterology 54: 172-179, 2007.

21. Nagano H, Miyamoto A, Wada $\mathrm{H}$, et al: Interferon- $\alpha$ and 5-fluorouracil combination therapy following palliative hepatic resection in patients with advanced hepatocellular carcinoma, portal venous tumor thrombus in the major trunk and multiple nodules. Cancer 110: 2493-2501, 2007.

22. The General Rules for the Clinical and Pathological Study of Primary Liver Cancer. 5th edition, Liver Cancer Study Group of Japan, 2008.

23. Oken MM, Creech RH, Tormey DC, et al: Toxicity and response criteria of the Eastern Cooperative Oncology Group. Am J Clin Oncol 5: 649-655, 1982.

24. Kaplan EL and Meier P: Nonparametric estimation from incomplete observations. J Am Stat Assoc 53: 457-481, 1958.

25. Ikai I, Arii S, Ichida T, et al: Report of the 16th follow-up survey of primary liver cancer. The Liver Cancer Study Group of Japan. Hepatol Res 32: 163-172, 2005. 
26. Yamakado K, Tanaka N, Nakatsuka A, et al: Clinical efficacy of portal vein stent placement in patients with hepatocellular carcinoma invading the main portal vein. J Hepatol 30: 660-668, 1999.

27. Cancer of the Liver Italian Program (CLIP) investigators: A new prognostic system for hepatocellular carcinoma: a retrospective study of 435 patients. Hepatology 28: 751-755, 1998.

28. Asahara T, Itamoto T, Katayama K, et al: Hepatic resection with tumor thrombectomy for hepatocellular carcinoma with tumor thrombi in the major vasculature. Hepatogastroenterology 46: 1862-1869, 1999.

29. Ohkubo T, Yamamoto J, Sugawara Y, et al: Surgical results for hepatocellular carcinoma with macroscopic portal vein thrombosis. J Am Coll Surg 191: 657-660, 2000.

30. Furuse J, Iwasaki M, Yoshino M, et al: Hepatocellular carcinoma with portal vein tumor thrombus: embolization of arterioportal shunts. Radiology 204: 787-790, 1997.

31. Lee HS, Kim JS, Choi IJ, et al: The safety and efficacy of transcatheter arterial chemoembolization in the treatment of patients with hepatocellular carcinoma and main portal vein obstruction: a prospective controlled study. Cancer 79: 2087-2094, 1997.

32. Poon RT, Ng KK, Lam CM, et al: Learning curve for radiofrequency ablation of liver tumors: prospective analysis of initial 100 patients in a tertiary institution. Ann Surg 239: 441-449, 2004.

33. Mazzaferro V, Regalia E, Doci R, et al: Liver transplantation for the treatment of small hepatocellular carcinomas in patients with cirrhosis. N Engl J Med 334: 693-700, 1996.

34. Poon RT, Fan ST, Ng IO and Wong J: Prognosis after hepatic resection for stage IVA hepatocellular carcinoma: a need for reclassification. Ann Surg 237: 376-383, 2003.

35. Ikai I, Yamaoka Y, Yamamoto Y, et al: Surgical intervention for patients with stage IV-A hepatocellular carcinoma without lymph node metastasis proposal as a standard therapy. Ann Surg 227: 433-439, 1998

36. Shi J, Lai ECH, Li N, et al: Surgical treatment for hepatocellular carcinoma with portal vein tumor thrombus. Ann Surg Oncol 17: 2073-2080, 2010.
37. Tazawa J,Maeda M, Sakai Y, et al: Radiation therapy in combination with transcatheter arterial chemoembolization for hepatocellular carcinoma with extensive portal vein involvement. J Gastroenterol Hepatol 16: 660-665, 2001.

38. Ishikura S, Ogino T, Furuse J, et al: Radiotherapy after transcatheter arterial chemoembolization for patients with hepatocellular carcinoma and portal vein tumor thrombus. Am J Clin Oncol 25: 189-193, 2002.

39. Minagawa M, Makuuchi M, Takayama T and Ohtomo K: Selection criteria for hepatectomy in patients with hepatocellular carcinoma and portal vein tumor thrombus. Ann Surg 233: 379-384, 2001.

40. Peng BG, He Q, Li JP and Zhou F: Adjuvant transcatheter arterial chemoembolization improves efficacy of hepatectomy for patients with hepatocellular carcinoma and portal vein tumor thrombus. Am J Surg 198: 313-318, 2009.

41. Nakamura M, Nagano H, Marubashi S, et al: A pilot study of combination chemotherapy of S-1, a novel oral DPD inhibitor, and interferon-alpha for advanced hepatocellular carcinoma with extrahepatic metastasis. Cancer 112: 1765-1771, 2008.

42. Llovet JM and Bruix J: Molecular targeted therapies in hepatocellular carcinoma. Hepatology 48: 1312-1327, 2008.

43. Yang ZF, Poon RT, Liu Y, et al: High doses of tyrosine kinase inhibitor PTK787 enhance the efficacy of ischemic hypoxia for the treatment of hepatocellular carcinoma: dual effects on cancer cell and angiogenesis. Mol Cancer Ther 5: 2261-2270, 2006.

44. Zhu AX, Sahani DV, Duda DG, et al: Efficacy, safety, and potential biomarkers of sunitinib monotherapy in advanced hepatocellular carcinoma: a phase II study. J Clin Oncol 27: 3027-3035, 2009.

45. Murakami M, Kobayashi S, Marubashi S, et al: Tyrosine kinase inhibitor PTK/ZK enhances the anti-tumor effects of interferon- $\alpha / 5$-fluorouracil therapy for hepatocellular carcinoma cells. Ann Surg Oncol 18: 589-596, 2011. 